\title{
Scaling functions in conical indentation of elastic-plastic solids
}

\author{
X. Y. Feng, T. C. Wang \\ State Key Laboratory of Nonlinear Mechanics, Institute of Mechanics, Chinese Academy of Sciences, \\ Beijing, China \\ Received 24 January 2007; Accepted 15 May 2007; Published online 17 July 2007 \\ (C) Springer-Verlag 2007 \\ Summary. The finite element method was used to simulate the conical indentation of elastic-plastic solids \\ with work hardening. The ratio of the initial yield strength to the Young's modulus $Y / E$ ranged from \\ 0 to 0.02 . Based on the calculation results, two sets of scaling functions for non-dimensional hardness $H / K$ \\ and indenter penetration $h$ are presented in the paper, which have closed simple mathematical form and can \\ be used easily for engineering application. Using the present scaling functions, indentation hardness and \\ indentation loading curves can be easily obtained for a given set of material properties. Meanwhile one can \\ use these scaling functions to obtain material parameters by an instrumented indentation load-displacement \\ curve for loading and unloading if Young's modulus $E$ and Poisson's ratio $v$ are known.
}

\section{Introduction}

Indentation experiments have been performed for nearly one hundred years for measuring the hardness of materials [1]. During the past decade, interest in indentation has increased significantly; first, because sample preparation is less elaborate than for other testing methods, and second, because of the noticeable improvement of indentation equipment and the need for measuring the mechanical properties of materials on small scales. With this improvement it is now possible to monitor with high precision both the load and the displacement of an indenter during indentation experiments in the micro-Newtons and nanometer ranges, respectively [2][4]. For nanoindentation, Berkovich tips, which are sharp three-sided pyramids, are commonly used. A load is applied to the tip, and the tip penetration into the material is measured. The contact stiffness between tip and the material tested is then determined either from the peak load or the initial slope of the unloading curves [5], [6]. Using the contact stiffness, hardness and Young's modulus may be calculated. But both methods depend on estimating the contact area under load, which is sometimes difficult, especially when "piling-up" occurs.

Correspondence: T. C. Wang, State Key Laboratory of Nonlinear Mechanics, Institute of Mechanics, Chinese Academy of Sciences, Beijing 100080, P. R. China

E-mail: tcwang@imech.ac.cn 
Recently, many attempts have been made to better understand indentation loading-curves. For example, the elastic and plastic properties may be computed utilizing a procedure suggested by Giannakopoulos and Suresh [7]. Finite element methods have been used to extract the mechanical properties of materials by matching the simulated loading and unloading curves with the experimentally determined ones [8], [9]. Also, finite element methods combined with dimensional analysis have been used to extract the mechanical properties of materials [10] and scaling relationships for indentation were determined [11], [12].

In this study, we use the finite element method to analyze the conical indentation in elasticplastic solids with work hardening. Based on the calculation results the scaling functions for the hardness and loading curves are presented, which are quite simple and used easily for engineering application.

\section{Finite element calculations and discussions}

The power law description is applied to the present analysis. The stress-strain curve of a power law hardening material under uniaxial tension is assumed to be given by

$\sigma=E \varepsilon, \quad$ for $\varepsilon \leq Y / E$,

$\sigma=K \varepsilon^{n} \quad$ for $\varepsilon \geq Y / E$,

where $E$ is Young's modulus, $Y$ is the initial yield stress, $K$ is the strength coefficient, and $n$ is the work-hardening exponent. To ensure continuity, we note $K=Y(E / Y)^{n}$. For most metals, $n$ has a value between 0.1-0.5 [13]. We further assume that the friction coefficient at the contact surface between the indenter and the solid is zero.

The illustration of conical indentation is shown in Fig. 1, where $a$ is the contact radius, $h_{c}$ is the contact depth, $h$ is the indenter displacement and $F$ is the force. The hardness can be evaluated by

$H=\frac{F}{\pi a^{2}}$.

In this Section we consider a three-dimensional, rigid, conical indenter of a given half angle, $\theta=68^{\circ}$, indenting normally into an elastic-plastic solid with work hardening. Finite-element calculations have been carried out using ABAQUS. Similar to the finite-element calculations [11], the large strain elasto-plastic feature of ABAQUS is used. In the finite-element model the

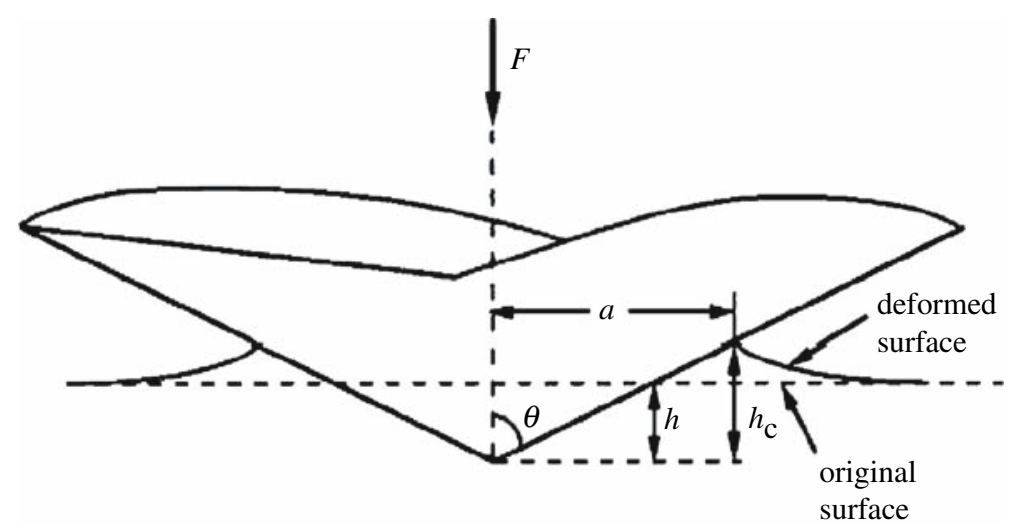

Fig. 1. Illustration of conical indentation 
indenter and solid were modelled as bodies of revolution to take advantage of the axisymmetry of the conical indentation. The indenter was modelled as a rigid body. The surface of the rigid indenter was defined using the ABAQUS feature of analytic rigid surface definition. The semiinfinite elastic-plastic solid was modelled using 14400 4-node bilinear axisymmetric quadrilateral elements. A fine mesh in the vicinity of the indenter and a gradually coarser mesh away from the indenter were used to ensure a high degree of numerical accuracy and a good representation of the semi-infinite solid. In the finite-element calculations we take $v=0.3$.

The non-dimensional hardness $H / K$ versus $Y / E$ is illustrated in Fig. 2, where the symbols represent the present finite-element calculation results for several values of $n$, and the solid lines represent the linear fitting results by the following functions:

$\frac{H}{K}=f_{0}+f_{1} \frac{Y}{E}$,

where $f_{0}$ and $f_{1}$ are the linear fit parameter values, which are shown with full squares in Fig. 3 and Fig. 4, respectively.

Figure 2 shows clearly that the linear fitting results are in good agreement with the finite element calculations in the case of $0.05<n \leq 0.5$. But the linear fitting results deviate from the finite element calculation results for the case $n \leq 0.05$ and $Y / E \leq 0.01$, that is because the accuracy of the finite element results is not enough. As we know, for a rigid-perfectly plastic solid, it is not easy to get high accuracy results for the finite element calculation. According to the theoretical result by Lockett [14], the ratio $H / Y$ is about 2.69 for a rigid-perfectly plastic solid, which is about $6 \%$ larger than the present finite calculation result. Hence the accuracy of the results for the finite element calculation is not enough and needs to be improved in the case of $n \leq 0.05$ and $Y / E \leq 0.01$. The present linear fitting results for $n=0$ are obtained according to the finite element calculations for $0.01 \leq Y / E \leq 0.02$ and Lockett's result. Meanwhile the present linear fitting results for $n=0.05$ are obtained according to the finite element calculations for $0.008 \leq Y / E \leq 0.02$.

In Figs. 3 and 4, the solid lines are the fitting results of linear fit parameters $f_{0}$ and $f_{1}$, respectively. One can clearly see that the linear parameter values are in good agreement with the

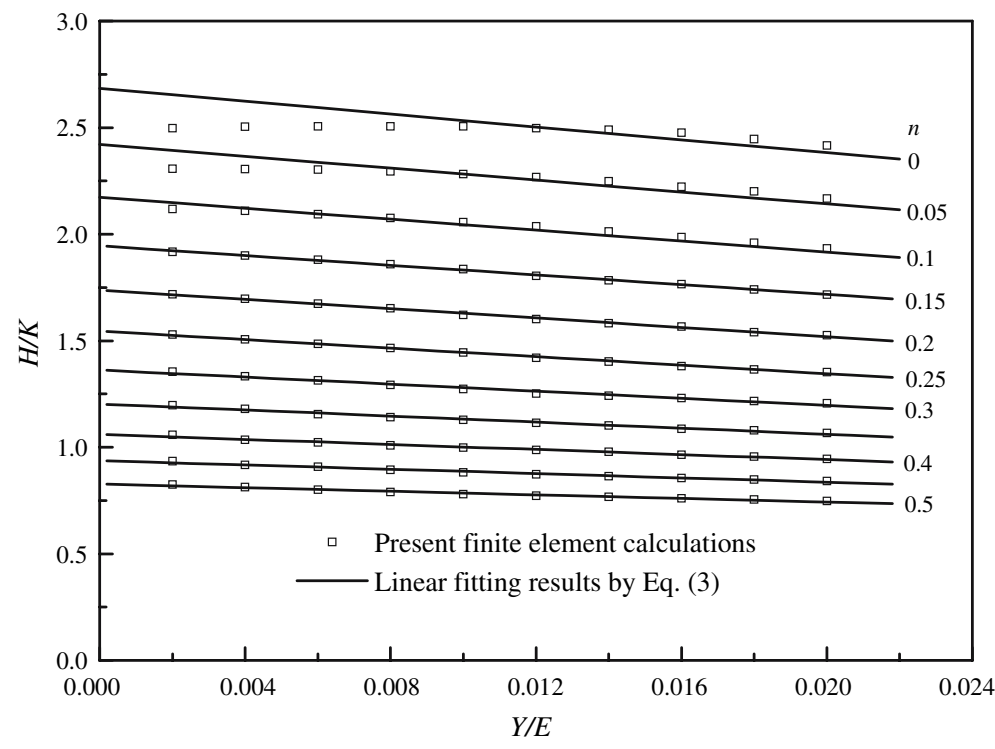

Fig. 2. The relationship between $H / K$ and $Y / E$ for several values of $n$ 


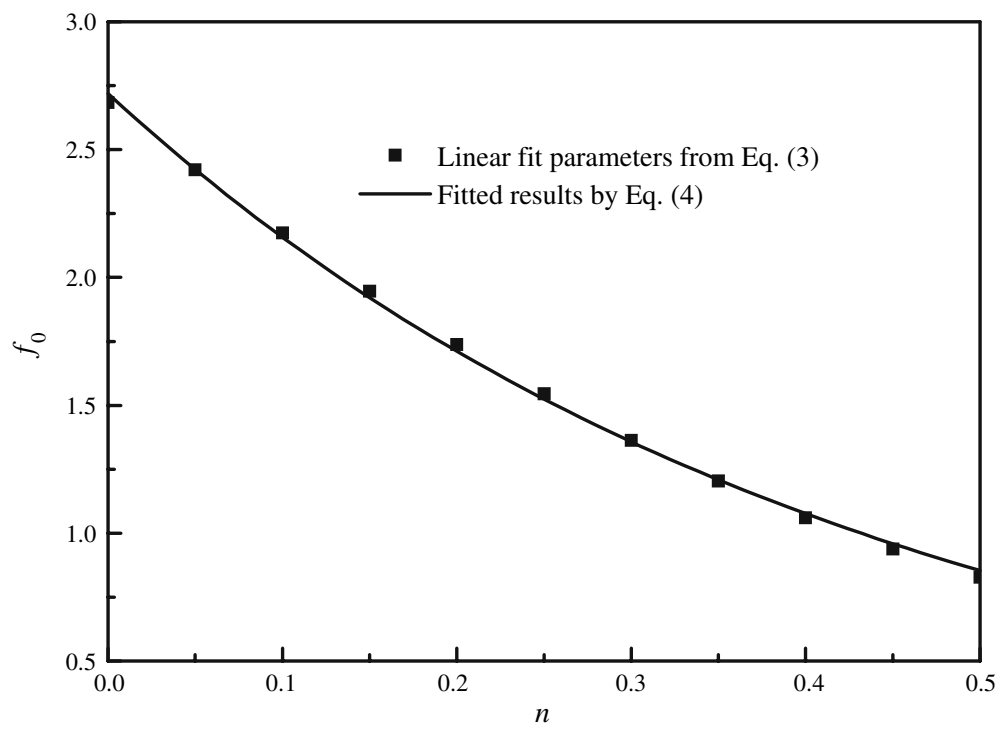

Fig. 3. The scaling function $f_{0}$ of indentation hardness

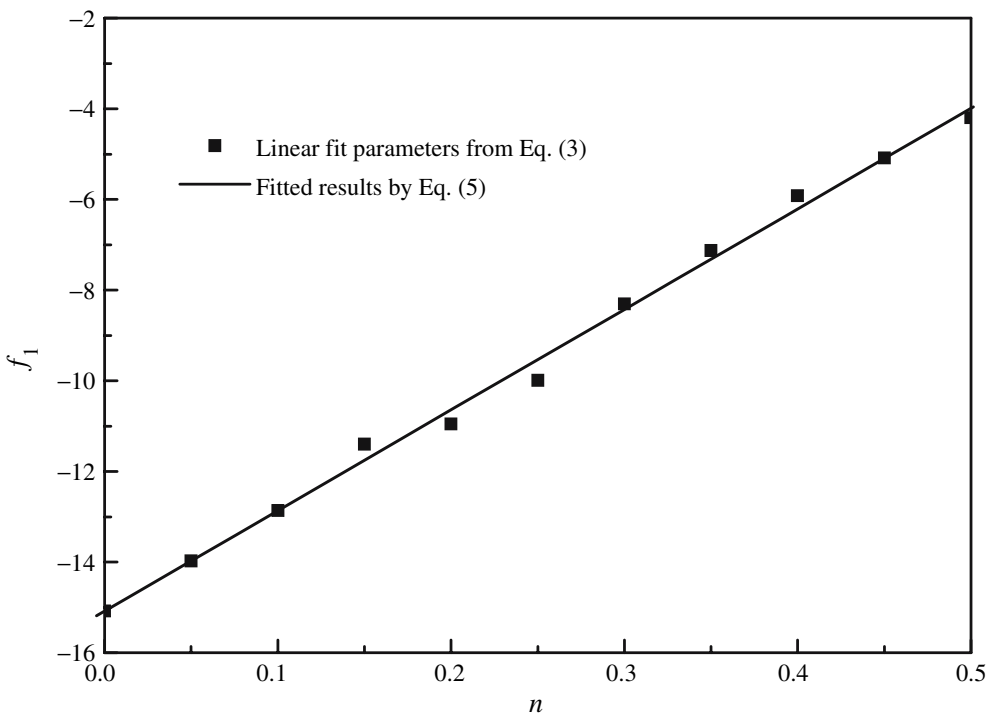

Fig. 4. The scaling function $f_{1}$ of indentation hardness

curve fitting results. By the curve fitting methods, the scaling functions of indentation hardness are given by

$f_{0}=2.720 \times(0.09857)^{n}$,

$f_{1}=-15.08+22.18 n$.

From Eqs. (3)-(5), it is obvious that the non-dimensional hardness $H / K$ is a function of the non-dimensional yield strength $Y / E$ and work hardening exponent $n$. Thus based on these functions $H / Y$ can be easily calculated for given values of $Y / E$ and $n$. 


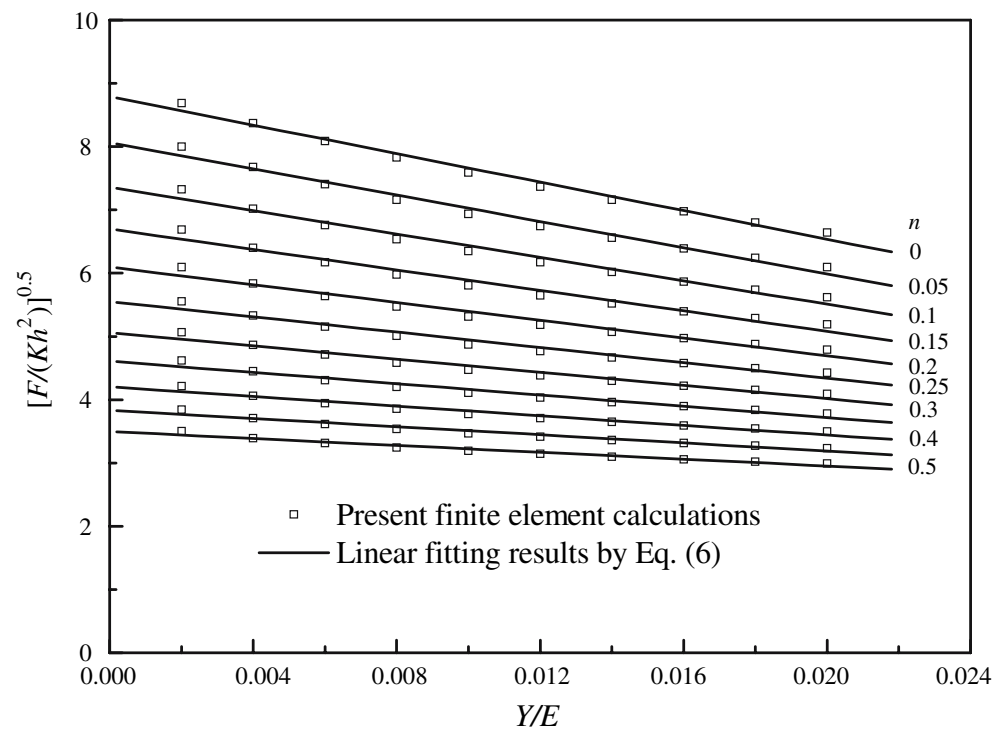

Fig. 5. The relationship between $\sqrt{P /\left(K h^{2}\right)}$ and $Y / E$ for several values of $n$

Figure 5 displays the relationship between the calculated $\sqrt{F /\left(K h^{2}\right)}$ and $Y / E$ for several values of $n$. The symbols represent the finite-element calculation results and the solid lines represent the linear fitting results by the following functions:

$\sqrt{\frac{F}{K h^{2}}}=a_{0}+a_{1} \frac{Y}{E}$

where $a_{0}$ and $a_{1}$ are the linear fit parameter values, which are shown with full squares in Fig. 6 and Fig. 7, respectively.

In Figs. 6 and 7, the solid lines are the fitting results of linear parameters $a_{0}$ and $a_{1}$, respectively. One can clearly see that the linear parameter values are in good agreement with the

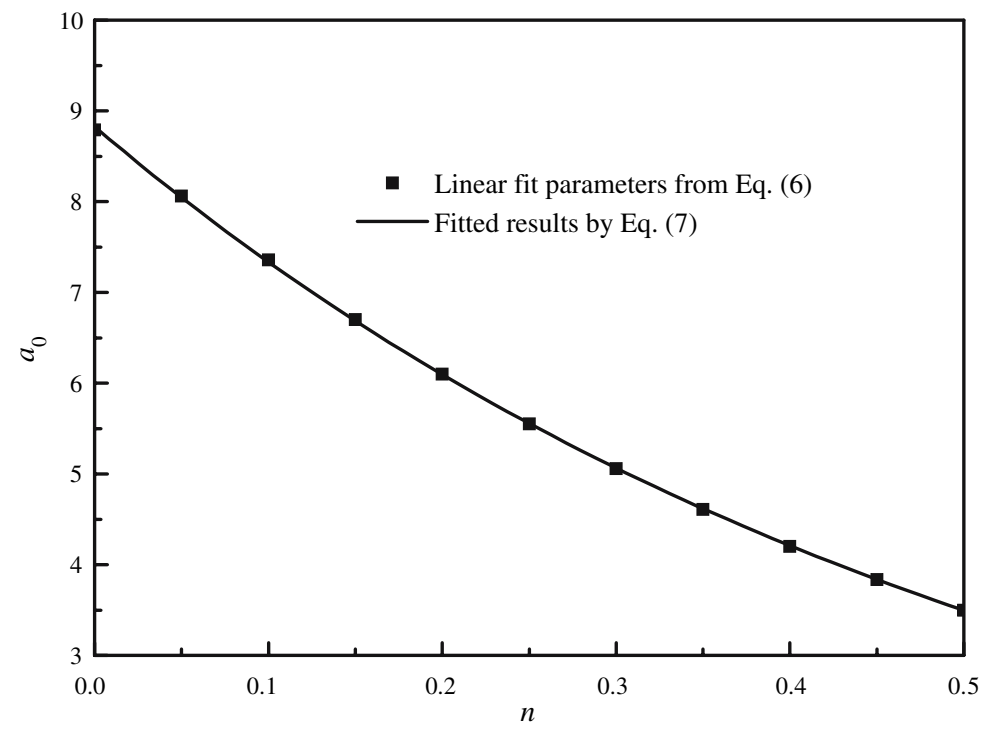

Fig. 6. The scaling function $a_{0}$ of indenter penetration 


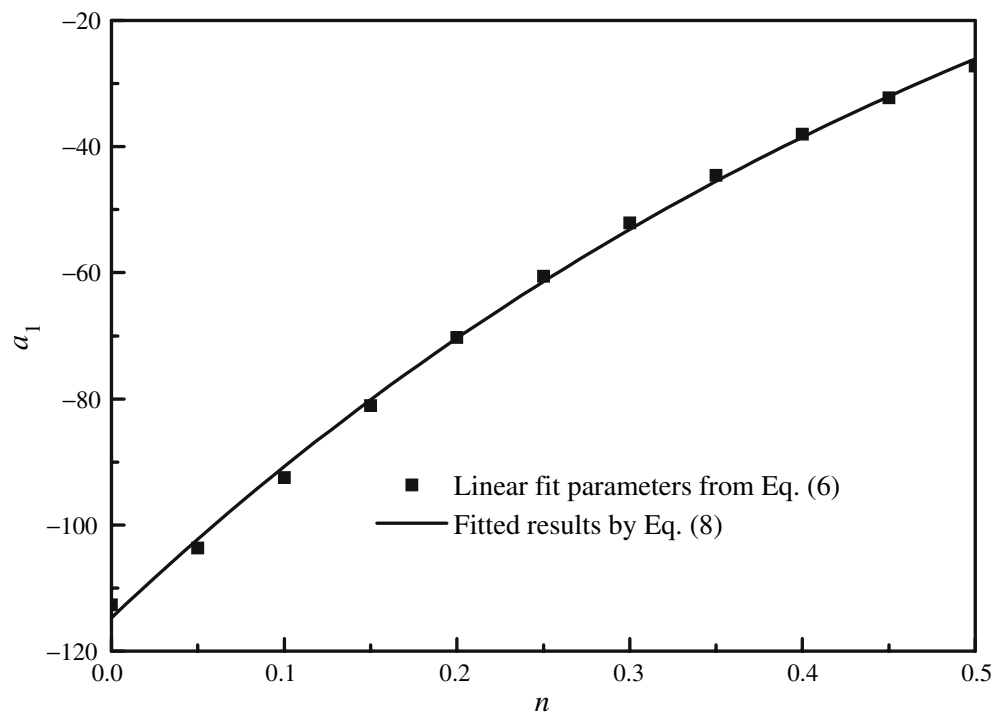

Fig. 7. The scaling function $a_{1}$ of indenter penetration

curve fitting results. By the curve fitting methods, the scaling functions of indenter penetration are given by

$a_{0}=8.826 \times(0.1571)^{n}$,

$a_{1}=43.22-158.0 \times(0.1925)^{n}$.

From Eqs. (6)-(8), it is obvious that, for loading, the force on the indenter is proportional to the square of the indenter displacement. Equation (6) can be rewritten as

$h=\sqrt{\frac{F}{K}} /\left(a_{0}+a_{1} \frac{Y}{E}\right)$.

The comparison of the predicted results for Eqs. (3)-(5) with the finite element calculations [11] is shown in Fig. 8. It is clearly shown that the predicted results agree well with the finite element calculation results [11] in the ranges of $0<Y / E \leq 0.1$ and $n \leq 0.5$.

\section{Property determination by indentation tests}

By finite element analyses of the sharp indenter, the following relationship has been derived for elastoplastic materials by Giannakopoulos and Suresh [7]:

$\frac{H}{E^{*}}=\frac{1}{d^{*}} \frac{h_{\max }-h_{r}}{h_{\max }}$,

where $h_{r}$ is the residual depth of penetration, $h_{\max }$ is the maximum penetration depth, $d^{*}=5$ for the Vickers pyramid indenter and $d^{*}=4.678$ for the Berckovich indenter, the conical indenter has results similar to Vickers or Berckovich depending on the included apex angle.

In Eq. (10), the effective elastic modulus, $E^{*}$, is commonly defined as

$E^{*}=\left(\frac{1-v^{2}}{E}+\frac{1-v_{i}^{2}}{E_{i}}\right)^{-1}$,

where $E_{i}$ and $v_{i}$ are Young's modulus and Poisson's ratio of the indenter. 


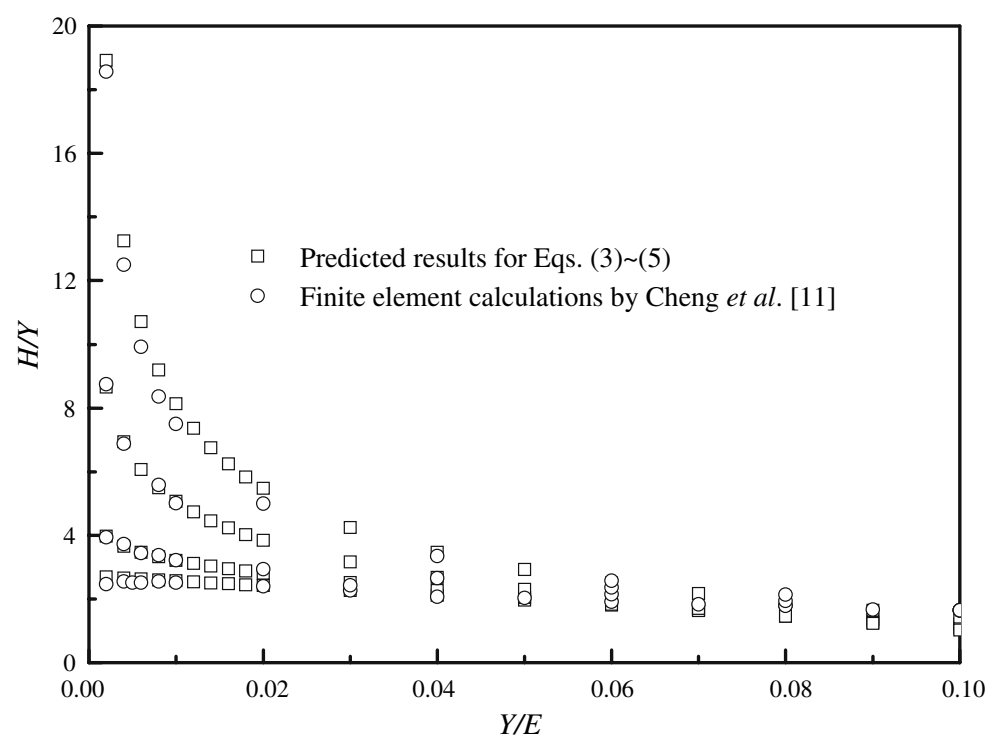

Fig. 8. The calculation results based on the scaling functions and the comparison with the finite element calculation results [11]

In the present finite element analyses the indenter is regarded as a rigid indenter, so the effective elastic modulus $E^{*}$ can be evaluated by

$E^{*}=\frac{E}{\left(1-v^{2}\right)}$.

Using Eqs. (3) and (10) we obtain

$\left(\frac{Y}{E}\right)^{1-n}\left(f_{0}+f_{1} \frac{Y}{E}\right)=\frac{E^{*}}{E} \frac{1}{d^{*}} \frac{h_{\max }-h_{r}}{h_{\max }}$.

Equation (6) or (9) can be rewritten as

$\left(\frac{Y}{E}\right)^{1-n}\left(a_{0}+a_{1} \frac{Y}{E}\right)^{2}=\frac{F}{E h^{2}}$.

A complete instrumented indentation $F-h$ curve obtained during loading and full unloading gives the following parameters: the loading curvature $C=F / h^{2}$, the maximum indentation depth $h_{\max }$ and the residual depth of penetration $h_{r}$. We assume that Young's modulus $E$ is known, initial yield stress $Y$ and work-hardening exponent $n$ can be determined using Eqs. (13) and (14).

Using the above method the initial yield stress $Y$ and the work-hardening exponent $n$ can be determined from the measured $F-h$ curve reported by Jayaraman et al. [15] for the Berckovich indentation of the 1070 steel.

According to Jayaraman et al. [15], the elastic modulus and Poisson's ratio are taken to be $207 \mathrm{GPa}$ and 0.3 , respectively. From the $F-h$ curve reported by Jayaraman et al. [15], the following values are extracted: $h_{\max }=0.733 \mu \mathrm{m}, h_{r}=0.567 \mu \mathrm{m}, C=186 \mathrm{GPa}$. From Eqs. (12)-(14), one obtains 
$\left(\frac{Y}{E}\right)^{1-n}\left(f_{0}+f_{1} \frac{Y}{E}\right)=0.05$,
$\left(\frac{Y}{E}\right)^{1-n}\left(a_{0}+a_{1} \frac{Y}{E}\right)^{2}=0.85$.

The above nonlinear equations can be solved by the zero point method of multiple variables [16]. Using this method one can get $Y=3.1 \mathrm{GPa}$ and $n=0.155$. The solution of Eqs. (15) and (16) for $Y$ and $n$ is unique in the ranges of $0<Y / E \leq 0.1$ and $n \leq 0.5$. Further one can obtain $K=Y(E / Y)^{n}=6 \mathrm{GPa}$. Figure 9 shows the comparison of the present calculated stress-strain curve for 1070 steel with the stress-strain curve predicted by Jayaraman et al. [15]. One can clearly see that both results agree well. Meanwhile the present calculated $F-h$ curve is in good agreement with the experimental curve [15], as shown in Fig. 10. Substituting these material parameters into Eq. (3), one gets the indentation hardness $H=10.35 \mathrm{GPa}$, which is about $8 \%$ higher than the value given by Jayaraman et al. [15].

\section{Concluding remarks}

The finite element calculations are performed for conical indentation in elastic-plastic solids with work hardening in which the non-dimensional initial yield strength $Y / E$ ranged from 0 to 0.02 and the hardening exponent $n$ ranged from 0 to 0.5 . Based on these calculation results, two sets of scaling functions for non-dimensional hardness $H / K$ and indenter penetration $h$ are presented in this paper, which are quite simple and used easily for engineering application. Using the scaling functions of indentation hardness, the indentation hardness can be easily evaluated, provided that the material parameters $Y / E$ and $n$ are known. The present analysis shows clearly that the pressure on the indenter is proportional to the square of the indenter displacement. Meanwhile one can use these scaling functions to get the material parameters $Y, K$ and $n$ based

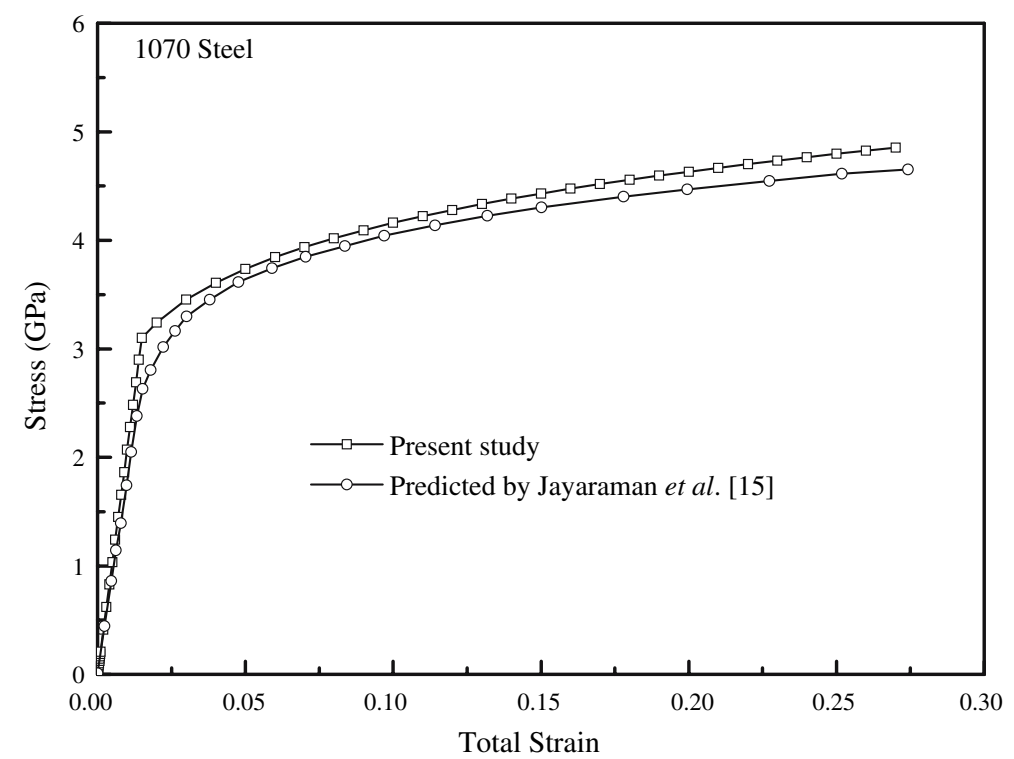

Fig. 9. The stress-strain curve of 1070 steel determined from the present study and comparison with the predicted curve [15] 


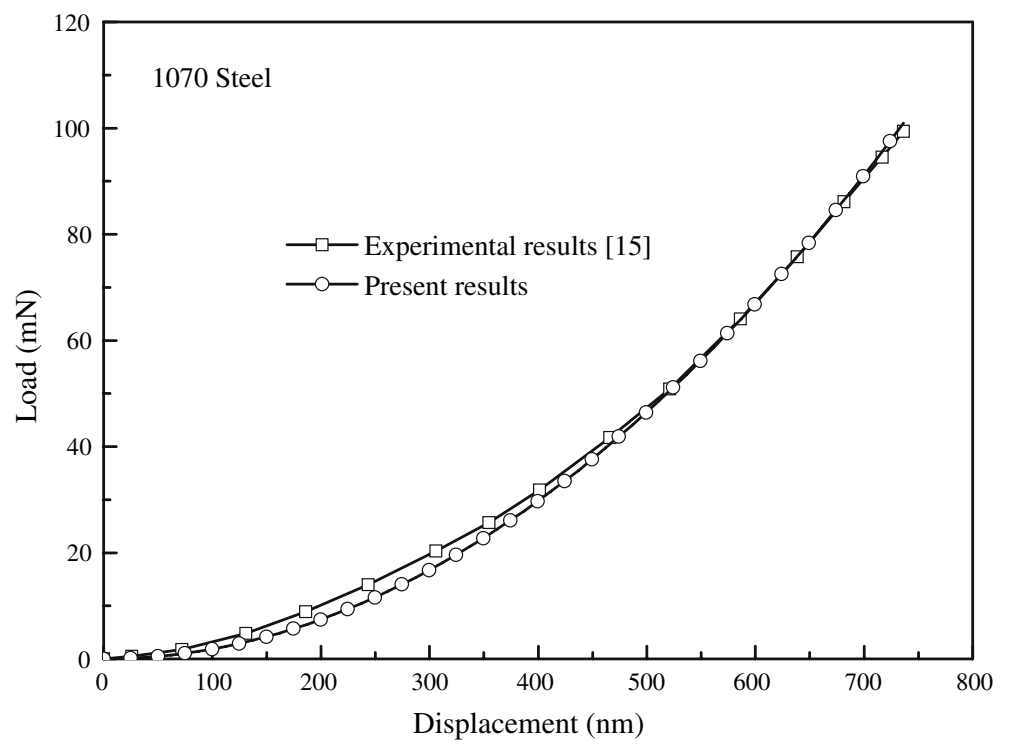

Fig. 10. The comparison of the present $F-h$ curve for 1070 steel with the experimental curve [15]

on the measured load-displacement curve and the measured full unloading curve at the maximum load if Young's modulus $E$ and Poisson's ratio $v$ are known.

Although the scaling functions of indentation hardness and indenter penetration have been derived for conical indentation, the same approach can be applied to indentation using pyramidal indenters and a conical indenter with different indenter angles. This approach is also not limited by the assumptions of rigid indenter and zero friction between indenter and solid. Using the present method the scaling functions for indentation hardness and the penetration which consider the effect of friction and indenter angle can be studied in the future.

\section{References}

[1] Tabor, D.: Indentation hardness: fifty years on - a personal view. Phil. Mag. A 74, 1207-1212 (1996).

[2] Pethica, J., Hutchings, R., Oliver, W.: Hardness measurement at penetration depths as small as 20-nm. Phil. Mag. A 48, 593-606 (1983).

[3] Stone, D., LaFontaine, W., Alexopoulos, P., Wu, T., Li, C.: An investigation of hardness and adhesion of sputter-deposited aluminum on silicon by utilizing a continuous indentation test. J. Mater. Res. 3, 141-147 (1988).

[4] Bhushan, B., Kulkarni, A., Bonin, W., Wyrobek, J.: Nanoindentation and picoindentation measurements using a capacitive transducer system in atomic force microscopy. Phil. Mag. A 74, 1117-1128 (1996).

[5] Doener, M., Nix, W.: A method of interpreting the data from depth-sensing indentation instruments. J. Mater. Res. 1, 601-609 (1986).

[6] Oliver, W., Pharr, G.: An improved technique for determining hardness and elastic modulus using load and displacement sensing indentation experiment. J. Mater. Res. 7, 1564-1583 (1992).

[7] Giannakopoulos, A., Suresh, S.: Determination of elastoplastic properties by instrumented sharp indentation. Scr. Mater. 40, 1191-1198 (1999).

[8] Bhattacharya, A., Nix, W.: Finite element simulation of indentation experiments. Int. J. Solids Struct. 24, 881-891 (1988). 
[9] Laurrsen, T., Simo, J.: A study of the mechanics of microindentation using finite elements. J. Mater. Res. 7, 618-626 (1992).

[10] Dao, M., Chollacoop, N., Vliet, K., Venkatesh, T., Suresh, S.: Computational modeling of the forward and reverse problems in instrumented sharp indentation. Acta. Mater. 49, 3899-3918 (2001).

[11] Cheng, Y., Cheng, C.: Scaling approach to conical indentation in elastic-plastic solids with work hardening. J. Appl. Phys. 84, 1284-1291 (1998).

[12] Cheng, Y., Cheng, C.: Scaling, dimensional analysis, and indentation measurements. Mater. Sci. Engng. Res. 44, 91-149 (2004).

[13] Dieter, G.: Mechanical metallurgy, 2nd ed. New York: McGraw-Hill 1976.

[14] Lockett, F.: Indentation of a rigid plastic material by a conical indenter. J. Mech. Phys. Solids 11, 345-355 (1963).

[15] Jayaraman, S., Hahn, G., Oliver, W., Rubin, C., Bastias, P.: Determination of monotonic stressstrain curve of hard materials from ultra-low-load indentation tests. Int. J. Solids Struct. 35, 365-381 (1998).

[16] Zhang, Z.: Mastering MATLAB 6.5, p.123. Beijing: BUAA Press 2003 (In Chinese). 